\title{
Antifungal effect of electrospun nanofibers containing cetylpyridinium chloride against Candida albicans
}

Valdirene Alves dos SANTOS(a) Patricia Verônica Aulestia VIERA(a) Adriano Marim de OLIVEIRA(b) Maria Helena Ambrosio ZANIN(b) Maria Aparecida BORSATTI(a)

(a) Department of Stomatology, School of Dentistry, Universidade de São Paulo - USP, São Paulo, SP, Brazil.

(b) Laboratory of Chemical Processes and Particle Technology, Instituto de Pesquisas Tecnológicas - IPT, São Paulo, SP, Brazil.

Declaration of Interests: The authors certify that they have no commercial or associative interest that represents a conflict of interest in connection with the manuscript.

Corresponding Author:

Valdirene Alves dos Santos

E-mail:valsantos@hotmail.com

DOI: 10.1590/1807-3107BOR-2014.vol28.0049

Submitted: Feb 03, 2013

Accepted for publication: Jun 02, 2014

Last revision: Aug 18, 2014

\begin{abstract}
It is known that cetylpyridinium chloride (CPC) has in vitro and in vivo antifungal action against Candida albicans, with advantages over other common antiseptics. A CPC delivery-controlled system, transported in polymer nanofibers (PVP/PMMA), was developed to increase the bioavailability of the drug in contact with the oral mucosa. The objectives of this study were to determine if CPC in nanofiber has antifungal action against C. albicans, and in what concentration it must be incorporated, so that the fraction released can yield an inhibitory concentration. The nanofiber was prepared by electrospinning, and sterilized with gamma irradiation. Nanofiber disks with $0.05 \%, 1.25 \%, 2.5 \%$ and $5 \%$ CPC, with $5 \%$ miconazole (MCZ) and with no drug, as well as filter paper disks with 5\% CPC, with 5\% MCZ and with no drug were used in this study. A Candida albicans suspension (ATCC 90028) was inoculated in Mueller-Hinton Agar plates. The disks were placed on the plates and the inhibition zone diameters were measured $48 \mathrm{~h}$ later. The nanopolymeric disks contracted in contact with the agar. All the concentrations of CPC incorporated in the nanofiber presented inhibitory action against C. albicans. Concentrations of $2.5 \%$ and $5 \%$ CPC presented a significant advantage over the nanofiber with no drug, proving the antifungal action of CPC. Under these experimental conditions, 5\% CPC has greater inhibitory action against $C$. albicans than $5 \% \mathrm{MCZ}$, both in nanofiber and in filter paper. A modification made in the polymer to decrease the contraction rate may allow a larger inhibition zone to be maintained, thereby increasing the clinical usefulness of the polymer.
\end{abstract}

Keywords: Nanofibers; Drug Delivery Systems; Administration, Topical; Mouthwashes; Candida.

\section{Introduction}

Candida albicans is a commensal organism that can be isolated from the gastrointestinal tract, as well as the oral and vaginal mucosa, in up to $80 \%$ of healthy individuals. Normally, it does not represent a problem; however, in certain cases it can cause the symptomatic infection of mucous membranes..$^{1,2}$ A change in the pathogenic state of the Candida species may occur after the oral cavity environment has been altered. ${ }^{3}$ The clinical manifestations of candidal infection in the mouth vary from acute pseudomembranous candidiasis to chronic atrophic candidal mucositis, ${ }^{4}$ including denture stomatitis (DS), which is the most prevalent oral infection involving the Candida species, ${ }^{5}$ and which occurs in about $15 \%$ and $65 \%$ of denture wearers. $1,5,6,7,8$ 
Currently, there are a variety of topical and systemic antifungal agents available for treating oral candidiasis. The topical antifungal agents most commonly used are nystatin and miconazole (MCZ), which have undesirable side effects and the risk of developing fungal resistance. ${ }^{9}$ The main adverse effects observed with nystatin are unpleasant taste, nausea and motion sickness. Miconazole may cause drug interactions, even in the case of topical use.,10

Mouthwashes containing antiseptics, such as chlorhexidine, cetylpyridinium chloride (CPC), triclosan and others, have been studied as alternatives for traditional antifungal agents. It has been shown that CPC has proven antifungal activity in vitro and in vivo, with advantages over triclosan, and chlorhexidine. ${ }^{11,12}$ The commercially available concentration of CPC $(0.05 \%)$ was used on cultured epithelial cells of human mucosa, and was found to reduce the adhesion of $C$. albicans by $80 \%$, showing efficacy similar to that of $0.2 \%$ chlorhexidine, which achieved a $77 \%$ reduction, and surpassed $0.045 \%$ triclosan, which reduced adhesion by only $20 \% .{ }^{13}$ Other advantages of CPC are the lower risk of developing microbial resistance and of causing drug interactions. However, the major cause of treatment failure with antiseptics, including $\mathrm{CPC}$, is the short time that these topical agents stay in the mouth in active concentrations (low substantivity), ${ }^{14}$ requiring application several times a day, ${ }^{4}$ a practice which could decrease patient adherence to treatment. Furthermore, CPC in low concentrations may not act against resistant fungi..$^{15}$ These issues promoted the development of a system of controlled CPC release, transported in polymer nanofibers (PVP/PMMA), in order to increase the bioavailability of the drug in contact with the oral mucosa, as a future scenario for CPC use in oral treatments.

The aim of this study was to determine whether CPC in nanofibers has antifungal action against $C$. albicans, and to establish the concentration of CPC that should be incorporated into these nanofibers (polymer: PVP/PMMA), so that the fraction released can attain the Minimum Inhibitory Concentration (MIC) against this yeast.

\section{Methodology}

Polymeric nanofiber disks composed of polyvinylpyrrolidone (PVP) Kollidon ${ }^{\circledR}$ 90F (BASF, Ludwigshafen, Germany) and Eudragit ${ }^{\circledR}$ RS100 ammonio methacrylate copolymer type B (PMMA) (Evonik Industries, Worms, Germany) were previously prepared by electrospinning. They were characterized in the Laboratory of Chemical Processes and Particle Technology (LPP) of the Instituto de Pesquisas Tecnológicas - IPT, São Paulo, SP, Brazil, and sterilized by Co60 gamma irradiation (EMBRARAD Ltda., Cotia, Brazil). The disk diffusion test for antifungal susceptibility analysis was performed in the laboratory of the Department of Prosthodontics, School of Dentistry, Universidade de São Paulo - USP, SP, Brazil.

\section{Disk diffusion test}

The disk diffusion method was performed according to the NCCSL Approved Guideline (M44-A). ${ }^{16}$ Polymeric nanofiber films previously made with PVP/PMMA solutions were cut into disks $19 \mathrm{~mm}$ in diameter, and subdivided into several groups:

1. Nanofiber disks containing CPC (Sigma-Aldricht Corp., Saint Louis, USA) in the following concentrations: $0.05 \%, 1.25 \%, 2.5 \%$ and $5 \%$ (each $\mathrm{n}=6$ )

2. Nanofiber disks with no drug $(\mathrm{n}=15)$

3. Nanofiber disks containing $5 \% \mathrm{MCZ}$ (SigmaAldricht Corp., Saint Louis, USA) $(n=6)$

4. Whatman ${ }^{\circledast} 40$ Filter paper disks (GE Healthcare Life Sciences, Pittsburgh, USA) with 5\% CPC ( $n=15)$

5. Whatman ${ }^{\circledast} 40$ Filter paper disks (GE Healthcare Life Sciences, Pittsburgh, USA) with 5\% MCZ ( $\mathrm{n}=9$ )

6. Whatman ${ }^{\circledast} 40$ Filter paper disks (GE Healthcare Life Sciences, Pittsburgh, USA) with no drug $(n=4)$

\section{Inoculum preparation}

Candida albicans (ATCC 90028) were subcultured onto Sabouraud Dextrose Agar (DIFCO BD Diagnostic Systems, Sparks, USA), at $35^{\circ} \mathrm{C}\left( \pm 2{ }^{\circ} \mathrm{C}\right)$, to ensure purity and viability. Colonies were picked with a sterile bacteriological loop, and suspended in $5 \mathrm{ml}$ of sterile saline $0.145 \mathrm{~mol} / \mathrm{L}(8.5 \mathrm{~g} / \mathrm{L} \mathrm{NaCl}, 0.85 \%$ saline). The resulting suspension was vortexed for $15 \mathrm{~s}$ and the cell density was adjusted visually to obtain an equivalent transmittance of $0.5 \mathrm{McFarland}$ Standard at a 530nm wavelength. This procedure provided a standard yeast suspension containing 1 $x 10^{6}$ to $5 \times 10^{6}$ cells per $\mathrm{mL}$. 


\section{Inoculation of test plates and placement of disks on inoculated plates}

Agar Mueller-Hinton (Sigma-Aldricht Corp., Saint Louis, USA) plates were inoculated with the previously prepared suspension of Candida albicans. A sterile cotton swab was dipped into the suspension. The swab was then rotated several times and pressed firmly against the inside wall of the tube above the fluid level to remove excess fluid from the swab. The dried surface of a sterile Mueller-Hinton Agar plate was inoculated by streaking the swab evenly over the entire agar surface three times, rotating the plate approximately $60^{\circ}$ each time to ensure even distribution of the inoculum. In the final step, the rim of the agar was swabbed. The inoculated plates were left undisturbed for about five minutes to allow excess moisture to be absorbed, and the disks were then placed on the surface of the inoculated agar plate (inside a laminar flow chamber) using sterile tweezers to avoid contamination. All the disks were pressed down gently to ensure their complete contact with the agar surface. A distance of $30 \mathrm{~mm}$ between one disk and the other (center to center of the disks) and $15 \mathrm{~mm}$ from the edge of the plate were maintained to prevent the inhibition zones from overlapping.

\section{Reading the plates and interpreting the results}

After 48 hours of incubation at $35^{\circ} \mathrm{C}$, the inhibition zone diameters were measured with a caliper (in millimeters), after examining the uniformity of the fungal growth and checking for the presence of any sign of contamination. Inhibition zones were considered as those where no colonies were observed by visual inspection.

\section{Statistics}

Statistical analysis was performed using the ANOVA test with a variation factor (drug) and an auxiliary Tukey test, $p<0.05$ using the GraphPad Prism ${ }^{\circledR} 5.0$ (GraphPad Software, La Jolla, USA) software program.

\section{Results}

Nanofiber disks in contact with the agar showed contraction (final diameter smaller than $19 \mathrm{~mm}$ ). The nanofiber disk contraction for both MCZ and CPC was almost twice as great as that of the nanofiber disks with no drugs (38.9\% and $18.9 \%$, respectively). Filter paper disks underwent no contraction. Despite the nanofiber disk contraction, there was inhibition zone formation with all the concentrations tested in this study (Figure). The difference between the inhibition zone diameter and the final diameter of the disk after contraction was considered for interpretation of the results. The 5\% CPC concentration in nanofibers showed a slightly better action than the $5 \%$ CPC in filter paper disks $(8.2$ and $7.1 \mathrm{~mm}$ of inhibition, respectively), but this difference was not statistically significant $(p>0.05)$. The nanofibers with no drug also showed an inhibition zone (3.8 $\mathrm{mm}$ ); however, $5 \%$ and $2.5 \% \mathrm{CPC}$ in the nanofiber showed a significantly higher level of activity than the nanofiber alone $(p<0.01$ and $p<0.05$, respectively). The $5 \%$ and $2.5 \%$ CPC concentration showed better antifungal action against $C$. albicans than $5 \% \mathrm{MCZ}$, both in nanofiber disks $(p>0.05)$ and filter paper disks $(p<0.01)$. The inhibition zones produced by nanofiber disks containing $5 \% \mathrm{MCZ}$ were slightly larger $(p>0.05)$ than the inhibition zones produced by filter paper disks containing the same drug. (Figure 1) The filter paper disks with no drug did not show any inhibition zone.

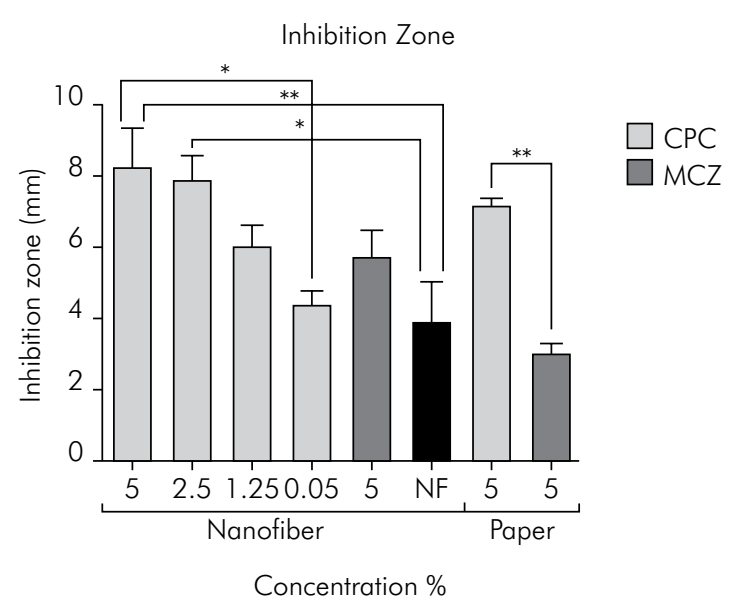

Figure. Histogram representing the mean \pm SE values of the difference between the inhibition zone diameter and the final diameter of the disk in the susceptibility test against Candida albicans. Statistical results (ANOVA and Tukey Test) are also shown: ${ }^{*} p<0.05,{ }^{* *} p<0.01$. CPC: cetylpyridinium chloride. MCZ: miconazole. NF: nanofiber with no drug. 


\section{Discussion}

In this study, the proposal was to use polymers (PVP/PMMA) in the form of nanofibers as a drug delivery system for cetylpyridinium chloride, for the purpose of treating oral candidiasis. Several different polymers are already used in formulations for drug delivery systems. Eudragit ${ }^{\circledR}$ RS100 was one of the compounds used in this study. It has been used successfully in systems for intestinal release of drugs ${ }^{17,18}$ and in transdermal drug delivery. ${ }^{19}$ The nanofibers presented in this study were evaluated to prove the efficacy of Eudragit ${ }^{\circledR}$ RS100 against C. albicans, by determining whether these nanofibers were capable of delivering CPC in an adequate concentration to provide inhibitory action against this microorganism for an extended period of time. The effect of CPC against $C$. albican $\mathrm{s}^{11,12,20}$ and in dental plaque control $^{21,22}$ has already been reported in the literature, in several studies, but CPC has never been used with this type of polymer. In regular mouthwashes, the concentration of CPC is $500 \mu \mathrm{g} / \mathrm{mL}(0.05 \%)$, which is 100 times the MIC for C. albicans found in some studies, ${ }^{15,23}$ however, these concentrations are rapidly diluted in the mouth, and attain ineffective concentrations that could also lead to the development of fungal resistance. ${ }^{15}$ For these reasons, mouthwashes need to be used several times a day, ${ }_{1}^{4}$ leading to lower patient adherence to treatment. We wanted to offset this disadvantage by making these nanofibers with CPC available to the population, as an alternative that would offer greater market availability, lower cost, and a satisfactory therapeutic outcome.

The results of the present study indicate that the antifungal action of CPC remains effective, even when it is encapsulated in polymeric nanofibers (Figure 1). Although there was no statistically significant difference between the encapsulated versus the filter paper disk versions of CPC release, it was observed that the concentration of encapsulated CPC has to be higher $(\geq 2.5 \%)$ than that used in common mouth rinses ( $0.05 \%)$ for this version to have an advantage over $5 \% \mathrm{CPC}$ in filter paper disks. Even if CPC must be provided in high concentrations, polymeric nanofibers could have the advantage of functioning as a controlled release system. Thus, there would be a lower frequency of antiseptic use, and application would be easier, result- ing in greater patient adherence to treatment, even in cases of incapacitated or hospitalized patients.

The nanofiber disks with no drugs also formed inhibition zones, showing that, in addition to the antifungal effect of CPC, there could also be an antifungal effect derived from the polymers composing the nanofiber. It has been observed that guanidine and amine polymethacrylates have an antifungal effect against $C$. albicans. ${ }^{24}$ This may occur because these polymers mimic antimicrobial peptides (AMPs) that are part of the innate defense system of the body, by acting on microorganism cell walls or membranes. Moreover, these polymers display a selective activity toward microorganisms versus mammalian cells. ${ }^{24,25}$ In addition to the chemical action of CCP and polymers, there may exist a mechanical action that causes yeast death by entrainment or occlusion.

The period of time analyzed in this study ( 48 hours) is longer than the periods evaluated in similar studies, and is one that could be used clinically, considering that patients usually brush their teeth and sanitize their prostheses at least once a day. In a study published in 1990, a multilayer bioadhesive lozenge containing CPC for slow administration was developed, and showed effective levels of drug release against $C$. albicans for only three hours. ${ }^{26}$ More recently, a buccoadhesive erodible disk of magnesium stearate, mannitol, sodium carboxy methyl cellulose DVP and hydroxypropyl methylcellulose $\mathrm{K} 4 \mathrm{M}$ was fabricated for CPC delivery, ${ }^{23}$ and showed diffusion of concentrations effective against $C$. albicans, lasting 6 hours.

The inhibitory zone produced by the disks containing $5 \% \mathrm{MCZ}$ was smaller than that produced by $5 \%$ CPC. This does not necessarily mean that MCZ has a weaker fungicidal effect than CPC. A possible explanation could be that $\mathrm{CPC}$ is released more quickly, because of its lower molecular weight (CPC: $339.98^{27}$ vs. MCZ: $416.12^{28}$ ).

The diameter of the nanofiber disks contracted when the disks were placed on the agar plates for the disk diffusion test. This leads us to imagine that similar contractions may occur when the film is applied on the oral mucosa, thereby decreasing the area of drug delivery. Other studies using polymers did not reveal the occurrence of this mechanical phe- 
nomenon; therefore, additional tests with modified polymers could help obtain films that undergo less contraction, thus ensuring drug delivery to a larger surface area of the oral mucosa for longer periods, and improving the clinical use of these drugs.

The methodology of this study had the limitation of evaluating only the antifungal activity and not the release pattern of the drug incorporated in the nanofiber, or the cytotoxicity of the polymer. Further complementary analysis (drug release profile, cytotoxicity, microbiological evaluation in biofilms) is required before clinical trials can be conducted with this technology, as an alternative therapy in treating oral fungal infections. In this study, we established a number of interesting correlations, which we were unable to exhaust in the discussion, due to current lack of evidence from similar trials with CPC in polymers against Candida albicans, but which will cer-

\section{References}

1. Budtz-Jørgensen E. Oral mucosal lesions associated with the wearing of removable dentures. J Oral Pathol. 1981 Apr;10(2):65-80. Review.

2. Kim J, Sudbery P. Candida albicans, a major human fungal pathogen. J Microbiol. 2011 Apr;49(2):171-7. doi: 10.1007/ s12275-011-1064-7. Epub 2011 May 3. Review.

3. Williams D, Lewis M. Pathogenesis and treatment of oral candidosis. J Oral Microbiol. 2011 Jan 28;3. doi: 10.3402/jom.v3i0.5771.

4. Samaranayake LP, Holmstrup P. Oral candidiasis and human immunodeficiency virus infection. J Oral Pathol Med. 1989 Dec;18(10):554-64. Review.

5. Shulman JD, Rivera-Hidalgo F, Beach MM. Risk factors associated with denture stomatitis in the United States.J Oral Pathol Med. 2005 Jul;34(6):340-6.

6. Budtz-Jörgensen E. Histopathology, immunology, and serology of oral yeast infections. Diagnosis of oral candidosis. Acta Odontol Scand. 1990 Feb;48(1):37-43. Review.

7. Gendreau L, Loewy ZG. Epidemiology and etiology of denture stomatitis. J Prosthodont. 2011 Jun;20(4):251-60. doi: 10.1111/j.1532-849X.2011.00698.x. Epub 2011; Apr 4. Review.

8. Newton AV. Denture sore mouth, a possible aetiology. Braz Dent J. 1962;112(1):357-60.

9. Muzyka BC, Somerman M. American Dental Association ADA/PDR. 4th ed. Montvale (NJ): Thompson PDR; 2006. Chapter 7, Antifungal and antiviral agents; p. 217-50.

10. Niimi M, Firth NA, Cannon RD. Antifungal drug resistance of oral fungi. Odontology. 2010 Feb;98(1):15-25. doi: 10.1007/ s10266-009-0118-3. Epub 2010 Feb 16. Review. tainly contribute to the future development of new alternatives for the treatment of other oral diseases.

\section{Conclusions}

The 5\% CPC concentration had a greater inhibitory action against $C$. albicans than $5 \% \mathrm{MCZ}$, under these experimental conditions. Despite the nanopolymeric disk contraction, CPC in PVP/PMMA nanofibers showed effective antifungal activity against Candida albicans (starting at concentrations of $0.05 \%$ ) for a longer period of time (48 hours) than the usual clinical period. Concentrations of $2.5 \%$ and higher present a significant advantage over nanofiber disks with no drug, thus proving the antifungal action of CPC. Modifying the polymer to decrease the contraction rate may make it possible to maintain a larger inhibition zone, thereby increasing the clinical usefulness of the polymer.

11. Giuliana G, Pizzo G, Milici ME, Musotto GC, Giangreco R. In vitro antifungal properties of mouthrinses containing antimicrobial agents. J Periodontol. 1997 Aug;68(8):729-33.

12. Giuliana G, Pizzo G, Milici ME, Giangreco R. In vitro activities of antimicrobial agents against s. Oral Surg Oral Med Oral Pathol Oral Radiol Endod. 1999 Jan;87(1):44-9. Erratum in: Oral Surg Oral Med Oral Pathol Oral Radiol Endod. 1999 Apr;87(4):524.

13. Pizzo G, Giuliana G, Milici ME, D'Angelo M. Effect of antimicrobial mouthrinses on the in vitro adhesion of Candida albicans to human buccal epithelial cells. Clin Oral Investig. 2001 Sep;5(3):172-6.

14. Powderly WG, Gallant JE, Ghannoum MA, Mayer KH, Navarro EE, Perfect JR. Oropharyngeal candidiasis in patients with HIV: suggested guidelines for therapy. AIDS Res Hum Retroviruses. 1999 Dec 10;15(18):1619-23. Review.

15. Edlind MP, Smith WL, Edlind TD. Effects of cetylpyridinium chloride resistance and treatment on fluconazole activity versus Candida albicans. Antimicrob Agents Chemother. 2005 Feb;49(2):843-5.

16. Clinical Laboratory Standard Institute (NCCLS). Method for Antifungal Disk Diffusion Susceptibility Testing of Yeasts; Approved Guideline. NCCLS document M44-A. Pensilvania; 2004.

17. Raval MK, Ramani RV, Sheth NR. Formulation and evaluation of sustained release enteric-coated pellets of budesonide for intestinal delivery. Int J Pharm Investig. 2013 Oct;3(4):20311. doi: 10.4103/2230-973X.121294. 
18. Akhgari A, Heshmati Z, Sharif Makhmalzadeh B. Indomethacin electrospun nanofibers for colonic drug delivery: preparation and characterization. Adv Pharm Bull. 2013;3(1):85-90. doi: 10.5681/apb.2013.014. Epub 2013 Feb 7.

19. Ofokansi KC, Kenechukwu FC, Ogwu NN. Design of novel miconazole nitrate transdermal films based on Eudragit RS100 and HPMC hybrids: preparation, physical characterization, in vitro and ex vivo studies. Drug Deliv [Internet] 2014 Jan 23. Available from: http://informahealthcare.com/ doi/abs/10.3109/10717544.2013.875604. doi:10.3109/10717544 .2013.875604. Epub ahead of print.

20. Regis RR, Della Vecchia MP, Pizzolitto AC, Compagnoni MA, Souza PP, Souza RF. Antimicrobial properties and cytotoxicity of an antimicrobial monomer for application in prosthodontics. J Prosthodont. 2012 Jun;21(4):283-90. doi: 10.1111/j.1532-849X.2011.00815.x. Epub 2012 Feb 19.

21. Hu D, Li X, Sreenivasan PK, DeVizio W. A randomized, doubleblind clinical study to assess the antimicrobial effects of a cetylpyridinium chloride mouth rinse on dental plaque bacteria. Clin Ther. 2009 Nov;31(11):2540-8. doi: 10.1016/j.clinthera.2009.11.004.

22. Costa X, Laguna E, Herrera D, Serrano J, Alonso B, Sanz M. Efficacy of a new mouth rinse formulation based on $0.07 \%$ cetylpyridinium chloride in the control of plaque and gingivitis: a 6-month randomized clinical trial. J Clin Periodontol. 2013 Nov;40(11):1007-15. doi: 10.1111/jcpe.12158. Epub 2013 Sep 11.
23. Ali J, Khar R, Ahuja A, Kalra R. Buccoadhesive erodible disk for treatment of oro-dental infections: design and characterisation. Int J Pharm. 2002 May 15;238(1-2):93-103.

24. Locock KE, Michl TD, Valentin JD, Vasilev K, Hayball JD, $\mathrm{Qu}$ Y, et al. Guanylated polymethacrylates: a class of potent antimicrobial polymers with low hemolytic activity. Biomacromolecules. 2013 Nov;14(11):4021-31. doi: 10.1021/bm401128r. Epub 2013 Oct 25.

25. Kuroda K, Caputo GA. Antimicrobial polymers as synthetic mimics of host-defense peptides. Wiley Interdiscip Rev Nanomed Nanobiotechnol. 2013 Jan-Feb;5(1):49-66. doi: 10.1002/wnan.1199. Epub 2012 Oct 17.

26. Collins AE, Deasy PB. Bioadhesive lozenge for the improved delivery of cetylpyridinium chloride. J Pharm Sci. 1990 Feb;79(2):116-9.

27. Center for Biotechnology Information [Internet]. PubChem Compound Database; CID=31239 [cited 2013 Sep 9]. Available from: http://pubchem.ncbi.nlm.nih.gov/summary/summary.cgi?cid=31239.

28. Center for Biotechnology Information [Internet]. PubChem Compound Database; CID=4189 [cited 2013 Sep 9]. Available from: http://pubchem.ncbi.nlm.nih.gov/summary/ summary.cgi?cid=4189. 